$\xi=$ 臬

\title{
Dynamics in the circular restricted three body problem with perturbations
}

\author{
Abdullah A. Ansari ${ }^{1}$ *, Mehtab Alam ${ }^{2}$ \\ ${ }^{1}$ Department of Mathematics, College of Science Al-Zulfi, Majmaah University, Kingdom of Saudi Arabia \\ ${ }^{2}$ HPS, Talimabad Campus, Sangam Vihar, New Delhi, India \\ *Corresponding author E-mail: a.ansari@mu.edu.sa
}

\begin{abstract}
This paper presents the dynamics in the restricted problem with perturbations i.e. the circular restricted three body problem by considering one of the primaries as oblate and other one having the solar radiation pressure and all the masses are variable (primaries and infinitesimal body). For finding the autonomized equations of motion, we have used the Meshcherskii transformation. We have drawn the libration points, the time series, the zero velocity curves and Poincare surface of sections for the different values of the oblateness and solar radiation pressure. Finally, we have examined the stability and found that all the libration points are unstable.
\end{abstract}

Keywords: Restricted Problem; Variable Masses; Oblate Body; Solar Radiation Pressure; Zero Velocity Curves; Poincare Surface of Sections.

\section{Introduction}

The restricted problem is the common model in the present age for the scientists in Mathematics and Astrophysics. Meshcherskii [1] explained the mechanics of bodies with variable mass. Szebehely [2] explained about the orbital motion, stability, and periodic orbits in his book "Theory of orbits". Chernikov [3] discussed the restricted three body problem (Sun-Planet-Particle) with the effects of solar radiation pressure. He found the six libration points and discussed the stability by Lyapunov's methods. Schuerman [4] explored the Roche potential with the effect of radiation pressure due to one component of a binary system. He demonstrated that the energy considerations of the modified Roche potential should have a greater tendency to form rings. Subbarao [5] investigated the stability of the triangular points of equilibrium points in the restricted three body problem with oblate primary. He observed that the triangular solutions form nearly equilateral triangles with the primaries and the range of the mass parameter which leads to stable triangular solution decreases. Bhatnagar $[6,7]$ studied the effect of oblateness in the potential on the libration points in the restricted three body problem and observed that the collinear libration points are unstable and for triangular points the range of stability decreases or increases. He also determined the mean motions and characteristic exponents at these points. Simmons [8] investigated the restricted 3-body problem with radiation pressure and observed that nine libration points exists, five in the plane of motion and four in the out of plane. Singh [9] studied the stability of triangular points in the generalized photogravitational restricted three body problem with oblate primary, and observed that the range of stability affected by radiation factor and oblateness. AbdulRaheem [10] investigated the stability of equilibrium points under the influence of the Coriolis and centrifugal forces together with the effects of oblateness and radiation pressure of the primaries. It is observed that the collinear points are unstable and the triangular points are conditionally stable depending on the radiation factor and oblateness. Singh [11] investigated the stability of equilibrium points in the restricted three body problem in which the masses of the luminous primaries vary isotropically in accordance with the unified Meshcherskii law. They found that the collinear points are unstable and the triangular points are conditionally stable in the autonomized system. Singh [12] investigated the positions and the linear stability of an infinitesimal body around the equilibrium points in the Robe's circular restricted three body problem, with the assumptions that the hydrostatic equilibrium figure of the first primary is an oblate spheroid and the second primary is an oblate body as well. He examined linear stability and found that the points near the center of the first primary are conditionally stable, while the others are unstable. Abouelmagd [13], [14] investigated the stability and periodic orbits in the restricted three body problem under the effects of oblateness and radiation pressure. He observed that the collinear points are unstable while triangular points are conditionally stable depending on the radiation pressure factor and oblateness. And also the elements of periodic orbits around equilibrium points are affected by oblateness. Abdullah [15] investigated the libration points and stability of the restricted four body problem with one of the primaries as oblate body and the infinitesimal body is taken as variable mass. Due to the oblateness, the triangular configuration becomes isosceles triangular configuration. They found the seven libration points out of which three are asymptotically stable and four are unstable. Mittal [16] investigated the stability of the Lagrangian solutions for the restricted four-body problem with variable mass. They found at most eight libration points in which two were collinear and rests were non-collinear and observed that all the libration points are unstable.

We have studied the dynamics in the restricted problem with perturbations in which the masses of the primaries as well as the mass of the infinitesimal body vary with time and one of the primaries is taken as oblate body and other one as radiation pressure. We have studied our problem in various sections. In the first section, we have introduced the problem. In the second section, we have evaluated the equations of motion of the infinitesimal variable mass in the cartesian form and in the autonomized form under the 
effects of the oblateness and radiation factor. In the third section, we have performed the numerical analysis (libration points, time series, zero velocity curves and Poincare surface of sections) for the different values of the parameters. In the fourth section, we have examined the stability of the libration points. And finally in the fifth section, we have concluded the problem. Our problem has many applications in this space age particularly in the Astrophysics.

\section{Equations of motion}

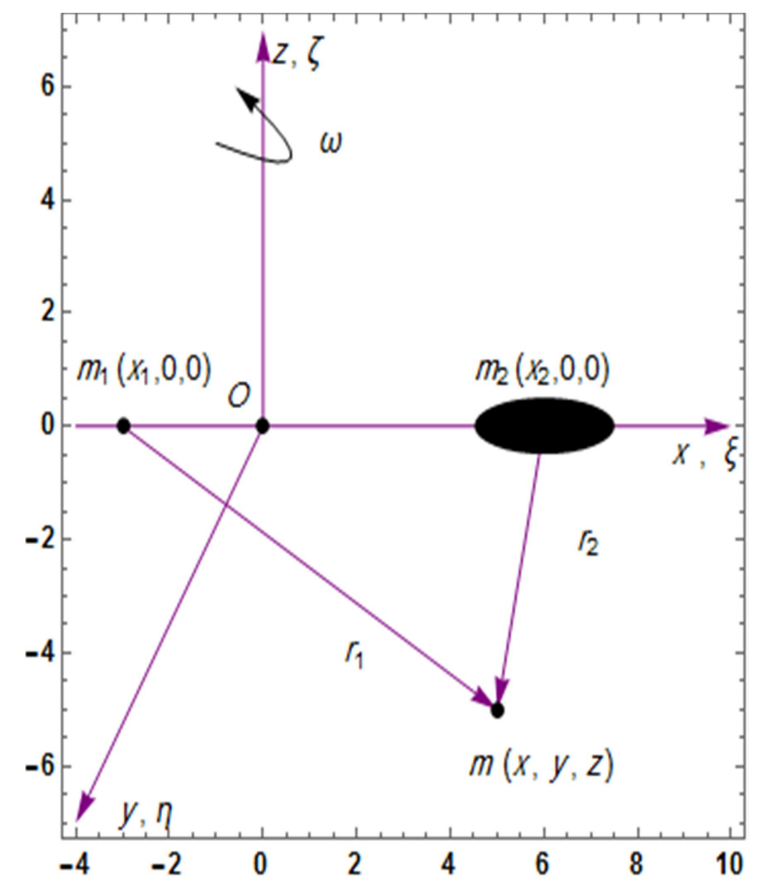

Fig. 1: Configuration of the Circular Restricted Three Body Problem when One of the Primaries, Oblate Body and other One as a Solar Radiation Pressure and All the Masses are Taken As Variable.

Let the masses of the primaries be $\mathrm{m}_{1}$ and $\mathrm{m}_{2}$ and the mass of the infinitesimal body be $\mathrm{m}$, all the masses vary with time. The primaries are revolving in the circular orbits around their center of mass which is considered as origin. The line joining these primaries is taken as $\mathrm{x}$-axis and the perpendicular line of $\mathrm{x}$-axis and passing through the origin is taken as $y$-axis. The line through the origin and perpendicular to the plane of motion of the primaries is taken as z-axis. Let us consider the synodic coordinate system, initially coincide with the inertial coordinate system, with angular velocity $\omega$ about $z$-axis. All the configuration are shown in figure1. Following the procedure of Abdullah [15], we can write the equations of motion of the infinitesimal variable mass in the circular restricted three body problem under the effects of oblateness and radiation pressure, when the variation of mass is non-isotropic and originates from one point as

$\frac{\dot{\mathrm{m}}}{\mathrm{m}}(\dot{\mathrm{x}}-\omega \mathrm{y})+\left(\ddot{\mathrm{x}}-\dot{\omega} \mathrm{y}-2 \omega \dot{\mathrm{y}}-\omega^{2} \mathrm{x}\right)=$

$$
\begin{aligned}
& -\frac{\mu_{1}\left(\mathrm{x}-\mathrm{x}_{1}\right) \mathrm{q}_{1}}{\mathrm{r}_{1}^{3}}-\frac{\mu_{2}\left(\mathrm{x}-\mathrm{x}_{2}\right)}{\mathrm{r}_{2}^{3}}-\frac{3 \mu_{2}\left(\mathrm{x}-\mathrm{x}_{2}\right) \sigma}{2 \mathrm{r}_{2}^{5}}, \\
& \frac{\dot{\mathrm{m}}}{\mathrm{m}}(\dot{\mathrm{y}}+\omega \mathrm{x})+\left(\ddot{\mathrm{y}}+\dot{\omega} \mathrm{x}+2 \omega \dot{\mathrm{x}}-\omega^{2} \mathrm{y}\right)=
\end{aligned}
$$$$
-\frac{\mu_{1} q_{1 y}}{r_{1}^{3}}-\frac{\mu_{2} y}{r_{2}^{3}}-\frac{3 \mu_{2} y \sigma}{2 r_{2}^{5}}
$$

$\frac{\dot{\mathrm{m}}}{\mathrm{m}} \dot{\mathrm{z}}+\ddot{\mathrm{z}}=-\frac{\mu_{1} \mathrm{z} \mathrm{q}}{\mathrm{r}_{1}^{3}}-\frac{\mu_{2} \mathrm{z}}{\mathrm{r}_{2}^{3}}-\frac{3 \mu_{2} \mathrm{z \sigma}}{2 \mathrm{r}_{2}^{5}}$,

Where, $r_{i}^{2}=\left(x-x_{i}\right)^{2}+y^{2}+z^{2},(i=1,2)$, are the distance from the primaries to the infinitesimal body,

$\mu_{i}=\frac{\mathrm{Gm}_{\mathrm{i}}}{\mathrm{m}_{1}+\mathrm{m}_{2}},(\mathrm{i}=1,2)$, are the masses of the primaries, $\mathrm{q}_{1}$ is the solar radiation factor, and oblateness factor $\sigma=\frac{\mathrm{a}_{1}^{2}-\mathrm{c}_{1}^{2}}{5 \mathrm{R}^{2}}, \mathrm{a}_{1}, \mathrm{c}_{1}$ are the semi axes of the oblate body, $\mathrm{R}$ is the distance between the primaries, angular velocity $\omega^{2}=\frac{G\left(m_{1}+m_{2}\right)}{R^{3}}\left(1+\frac{3}{2} \sigma\right), G$ is the gravitational constant.

Using Meshcherskii [2] transformation

$\begin{aligned} x & =\xi R(t), y=\eta R(t), z=\zeta R(t), \\ \frac{d t}{d \tau} & =R^{2}(t), r_{i}=\rho_{i} R(t), \quad(i=1,2),\end{aligned}$

The particular solutions of the Gylden-Meshcherskii problem

$\omega(t)=\frac{\omega_{0}}{R^{2}(t)}, x_{1}=\xi_{1} R(t), x_{2}=\xi_{2} R(t)$,

And the unified Meshcherskii law

$$
\begin{aligned}
& \mu(t)=\frac{\mu_{0}}{R(t)}, \mu_{1}(t)=\frac{\mu_{10}}{R(t)}, \mu_{2}(t)=\frac{\mu_{20}}{R(t)}, \\
& \mu(t)=\mu_{1}(t)+\mu_{2}(t) \\
& m=\frac{m_{0}}{R(t)}, R(t)=\sqrt{a t^{2}+2 b t+c}
\end{aligned}
$$

Where a, b,c, $\mu_{0}, \mu_{10}, \mu_{20}, \mathrm{~m}_{0}$ are constants.

$$
\begin{aligned}
& \dot{x}=\frac{\xi^{\prime}+(a t+b) \xi}{R(t)}, \ddot{x}=\frac{\xi^{\prime \prime}+\left(a c-b^{2}\right) \xi}{R^{3}(t)}, \\
& \dot{y}=\frac{\eta^{\prime}+(a t+b) \eta}{R(t)}, \ddot{y}=\frac{\eta^{\prime \prime}+\left(a c-b^{2}\right) \eta}{R^{3}(t)}, \\
& \dot{z}=\frac{\zeta^{\prime}+(a t+b) \zeta}{R(t)}, \ddot{z}=\frac{\zeta^{\prime \prime}+\left(a c-b^{2}\right) \zeta}{R^{3}(t)} .
\end{aligned}
$$

We transform the system (1) to the autonomous form

$$
\begin{aligned}
& \xi^{\prime \prime}-2 \omega_{0} \eta^{\prime}-(\mathrm{at}+\mathrm{b}) \xi^{\prime}=\frac{\partial \Omega}{\partial \xi}, \\
& \eta^{\prime \prime}+2 \omega_{0} \xi^{\prime}-(\mathrm{at}+\mathrm{b}) \eta^{\prime}=\frac{\partial \Omega}{\partial \eta}, \\
& \zeta^{\prime \prime}-(\mathrm{at}+\mathrm{b}) \zeta^{\prime}=\frac{\partial \Omega}{\partial \zeta} .
\end{aligned}
$$

Where, 
$\Omega=\frac{1}{2}\left((\mathrm{at}+\mathrm{b})^{2}+\omega_{0}^{2}-\Delta\right)\left(\xi^{2}+\eta^{2}\right)$

$+\frac{1}{2}\left((\mathrm{at}+\mathrm{b})^{2}-\Delta\right) \zeta^{2}-(\mathrm{at}+\mathrm{b}) \xi \eta$

$+\frac{\mu_{10 q_{1}}}{\rho_{1}}+\frac{\mu_{20}}{\rho_{2}}+\frac{\mu_{20} \sigma}{2 \rho_{2}^{3}}$,

$\rho_{\mathrm{i}}^{2}=\left(\xi-\xi_{\mathrm{i}}\right)^{2}+\eta^{2}+\zeta^{2}, \Delta=\mathrm{ac}-\mathrm{b}^{2}, \xi_{1}=\frac{-\mu_{20}}{\mu_{0}} \mathrm{R}, \xi_{2}=\frac{\mu_{10}}{\mu_{0}} \mathrm{R}$.

Dash (') is the differentiation w.r.t. $\tau$. Taking unit of mass, distance and time at initial time $\mathrm{t}_{0}$ such that

$\mu_{0}=1, \mathrm{R}=1, \mathrm{G}=1, \omega_{0}=1+\frac{3}{4} \sigma, \mathrm{at}_{0}+\mathrm{b}=\alpha_{1}$ (constant)

So, $\Delta=1-\mathrm{k}$, where $\mathrm{k}$ is constant of a particular integral of GyldenMeshcherskii problem.

Introducing the mass parameter v expressed as

$\frac{\mu_{10}}{\mu_{0}}=1-v, \frac{\mu_{20}}{\mu_{0}}=v, 0<v \leq \frac{1}{2}$,

Where $v$ is the ratio of the mass of the primaries to the total mass of the primaries.

Finally, the autonomized system (2) becomes

$$
\begin{gathered}
\xi^{\prime \prime}-2 \omega_{0} \eta^{\prime}-\alpha_{1} \xi^{\prime}=\frac{\partial \Omega}{\partial \xi}, \\
\eta^{\prime \prime}+2 \omega_{0} \xi^{\prime}-\alpha_{1} \eta^{\prime}=\frac{\partial \Omega}{\partial \eta}, \\
\zeta^{\prime \prime}-\alpha_{1} \zeta^{\prime}=\frac{\partial \Omega}{\partial \zeta} .
\end{gathered}
$$

Where,

$$
\begin{aligned}
& \Omega=\frac{1}{2}\left(\alpha_{1}^{2}+\mathrm{k}-1+\omega_{0}^{2}\right)\left(\xi^{2}+\eta^{2}\right) \\
& +\frac{1}{2}\left(\alpha_{1}^{2}+\mathrm{k}-1\right) \zeta^{2}-\alpha_{1} \xi \eta \\
& +\frac{\mathrm{k}(1-v) \mathrm{q}_{1}}{\rho_{1}}+\frac{\mathrm{kv}}{\rho_{2}}+\frac{\mathrm{kv} \sigma}{2 \rho_{2}^{3}}, \\
& \rho_{\mathrm{i}}^{2}=\left(\xi-\xi_{\mathrm{i}}\right)^{2}+\eta^{2}+\zeta^{2}, \xi_{1}=-v, \xi_{2}=1-v .
\end{aligned}
$$

\section{Numerical analysis}

\subsection{Libration points}

We can find the equilibrium points from the solution of the equations

$\Omega_{\xi}=0, \Omega_{\eta}=0, \Omega_{\zeta}=0$,

i.e.

$$
\begin{aligned}
& \left(\alpha_{1}^{2}+k-1+\omega^{2}\right) \xi-\alpha_{1} \eta- \\
& \frac{k q_{1}(1-v)(\xi+v)}{\left((\xi+v)^{2}+\eta^{2}+\zeta^{2}\right)^{3 / 2}}-\frac{k v(\xi+v-1)}{\left((\xi+v-1)^{2}+\eta^{2}+\zeta^{2}\right)^{3 / 2}} \\
& -\frac{3 k v(\xi+v-1) \sigma}{2\left((\xi+v-1)^{2}+\eta^{2}+\zeta^{2}\right)^{5 / 2}}=0, \\
& \left(\alpha_{1}^{2}+\mathrm{k}-1+\omega^{2}\right) \eta-\alpha_{1} \xi-\frac{\mathrm{kq}_{1}(1-v) \eta}{\left((\xi+v)^{2}+\eta^{2}+\zeta^{2}\right)^{3 / 2}} \\
& -\frac{k v \eta}{\left((\xi+v-1)^{2}+\eta^{2}+\zeta^{2}\right)^{3 / 2}}- \\
& \frac{3 k u \eta \sigma}{2\left((\xi+v-1)^{2}+\eta^{2}+\zeta^{2}\right)^{5 / 2}}=0 \text {, } \\
& \left(\alpha_{1}^{2}+k-1\right) \zeta-\frac{k q_{1}(1-v) \zeta}{\left((\xi+v)^{2}+\eta^{2}+\zeta^{2}\right)^{3 / 2}} \\
& -\frac{k v \zeta}{\left((\xi+v-1)^{2}+\eta^{2}+\zeta^{2}\right)^{3 / 2}}- \\
& \frac{3 k v \zeta \sigma}{2\left((\xi+v-1)^{2}+\eta^{2}+\zeta^{2}\right)^{5 / 2}}=0,
\end{aligned}
$$

Here, we have shown the libration points graphically and found at most nine libration points in both $(\xi, \eta)$ plane (Fig. 2, Fig. 3 and Fig. 4$)$ and $(\xi, \zeta)$ plane (Fig. 5 and Fig. 6$)$. In the $(\xi, \eta)$ plane, we have found seven libration points when there were no effects of oblateness and radiation pressure (Fig. 2). After taking the effect of oblateness only, we have found nine libration points (Fig. 3) and considering the effect of solar radiation pressure only, we have found seven libration points which are moving towards the origin (Fig. 4). In the $(\xi, \zeta)$ plane, we have found five libration points in all the cases but after considering the effects of oblateness and solar radiation pressure, we have found that the libration points are moving towards the origin (Fig. 5, Fig. 6).

$$
(\xi, \eta)-\text { plane } \quad \alpha_{1}=0.2, k=0.4, v=0.019
$$

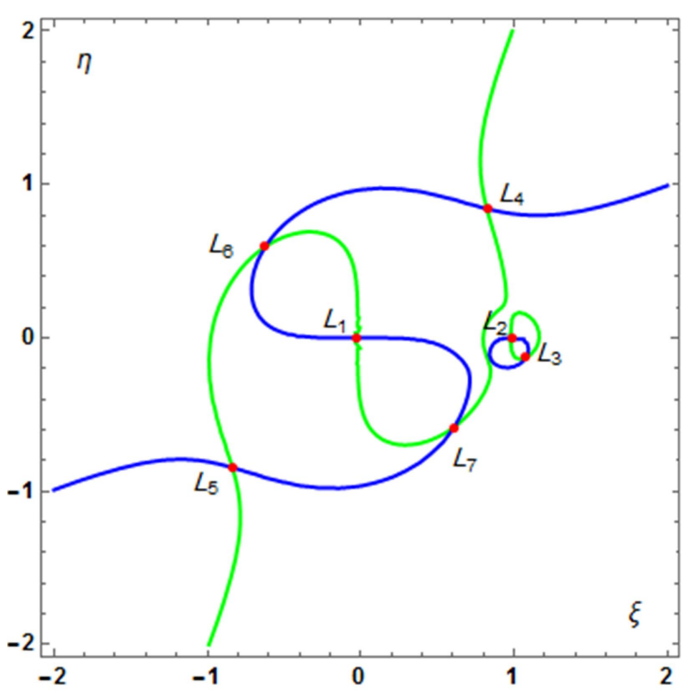

Fig. 2: Locations of equilibrium points at $\sigma=0, \mathrm{q}_{1}=1$. 


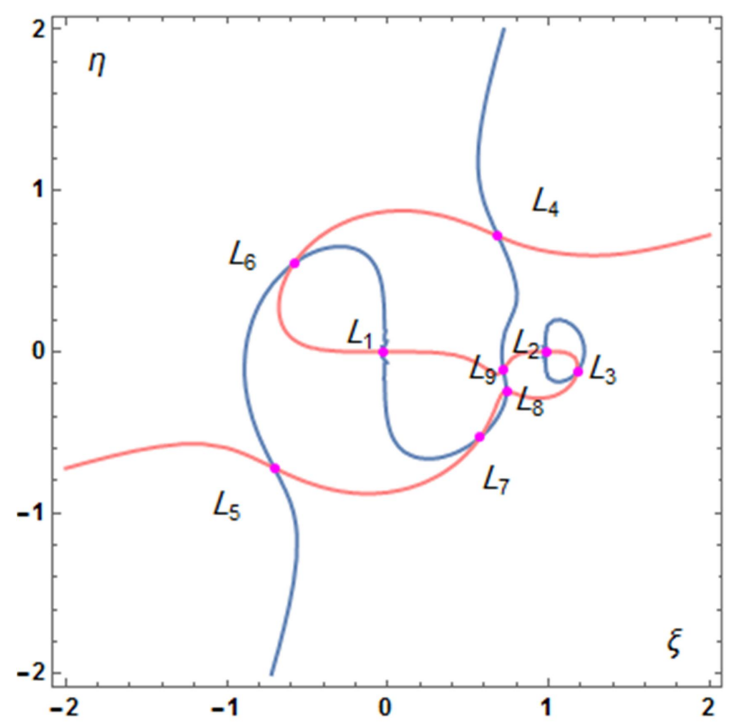

Fig. 3: Locations of Equilibrium points at $\sigma=0, \mathrm{q}_{1}=1$.

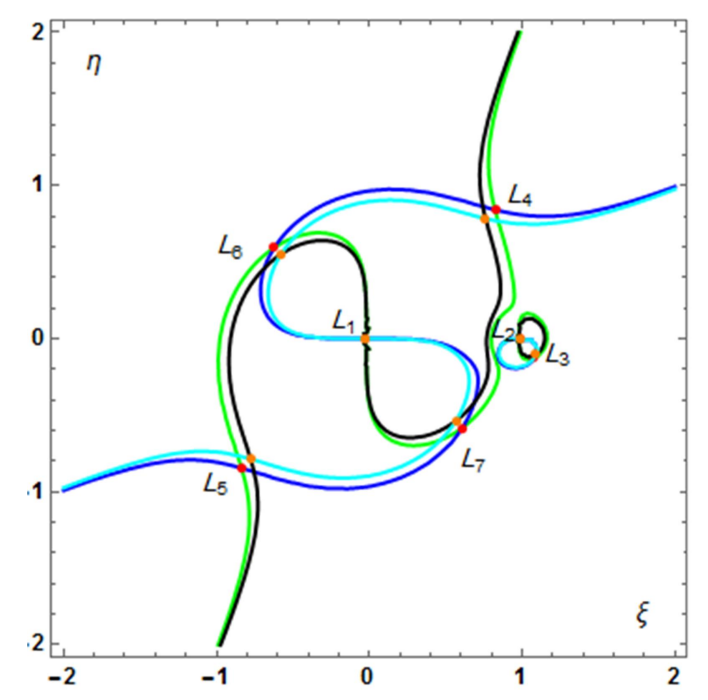

Fig. 4: Locations of Equilibrium points at $\sigma=0,\left\{\mathrm{q}_{1}=1(\operatorname{Red}), \mathrm{q}_{1}=0.8(\right.$ Orange $\left.)\right\}$

ii)

$(\xi, \zeta)$-plane $\quad \alpha_{1}=0.2, \mathrm{k}=0.4, \quad v=0.019$

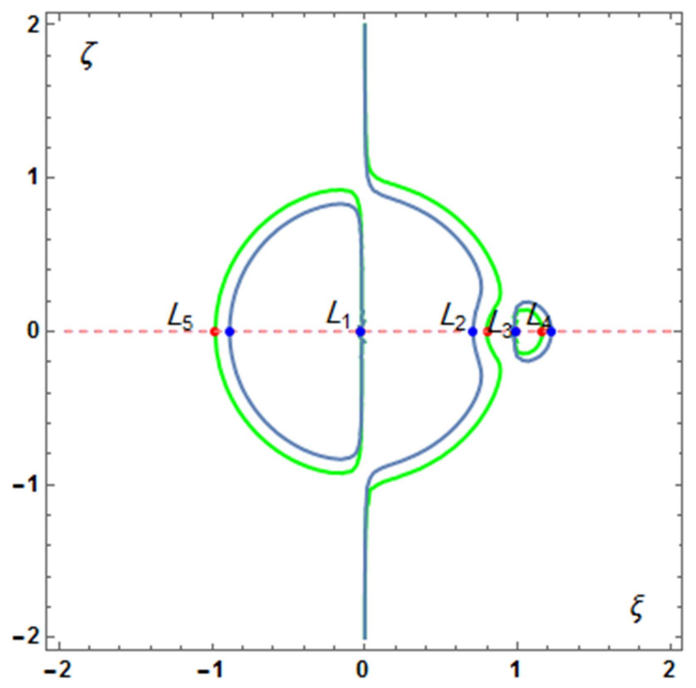

Fig. 6: Locations of Equilibrium points at $\sigma=0,\left\{\mathrm{q}_{1}=1(\operatorname{Red}), \mathrm{q}_{1}=0.8(\right.$ Orange $\left.)\right\}$

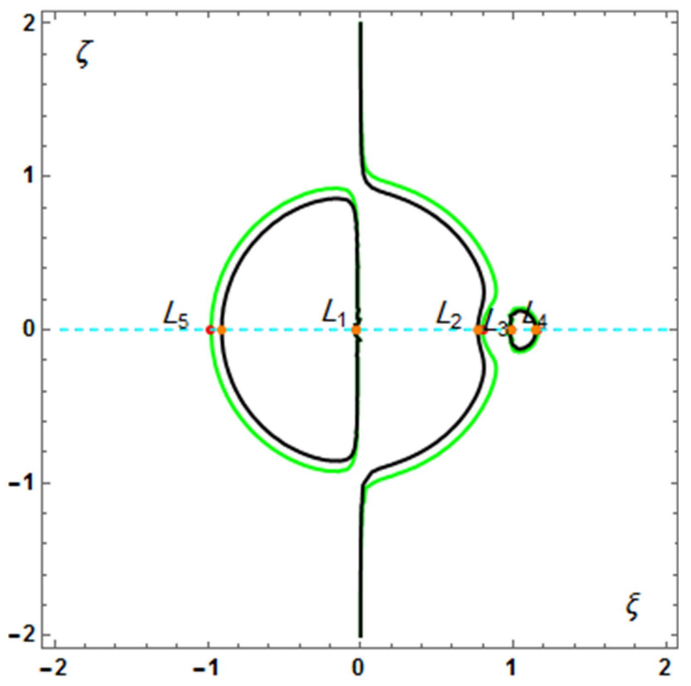

Fig. 6: Locations of Equilibrium points at $\sigma=0,\left\{\mathrm{q}_{1}=1(\operatorname{Red}), \mathrm{q}_{1}=0.8(\right.$ Orange $\left.)\right\}$.

\subsection{Time series}

We have drawn the time series in between $(\tau, \xi)$ and $(\tau, \eta)$, and observed that when we are considering the effects of oblateness and solar radiation pressure, they have shifted by some phase angles in all the series (Figs. 7, 8, 9, 10).

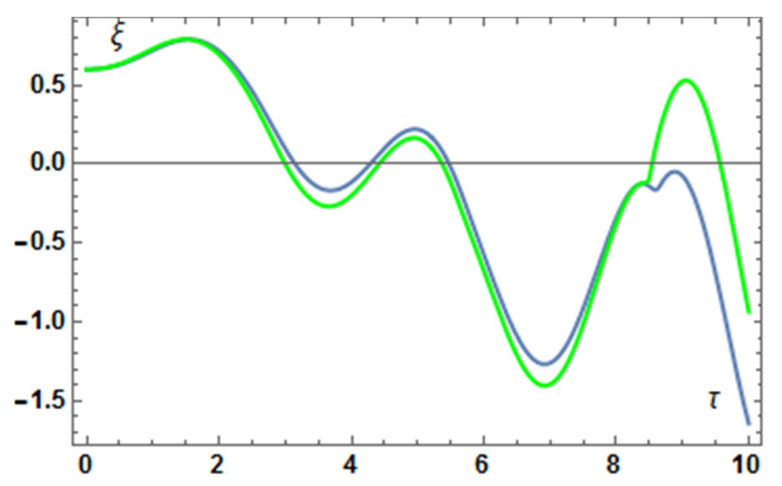

Fig.7: Time Series for $\mathrm{q}_{1}=1,\{\sigma=0$ (Magneta $), \sigma=0.1($ Green $\left.)\right\}$.

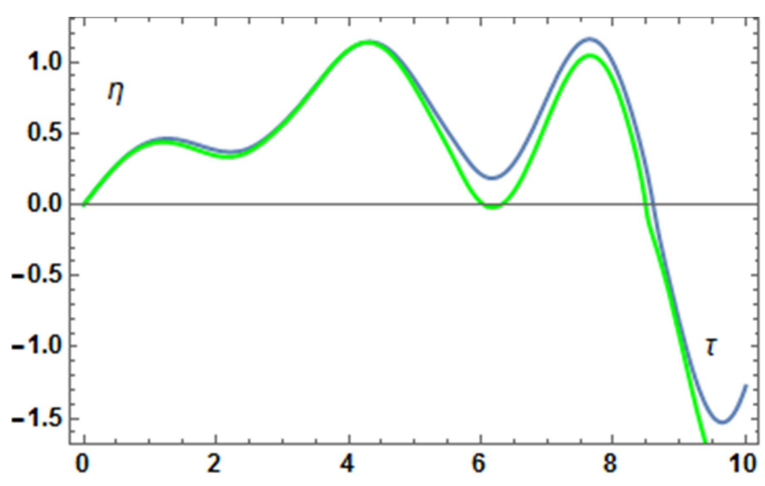

Fig.8: Time Series for $\mathrm{q}_{1}=1,\{\sigma=0$ (Magneta $), \sigma=0.1($ Green $\left.)\right\}$. 


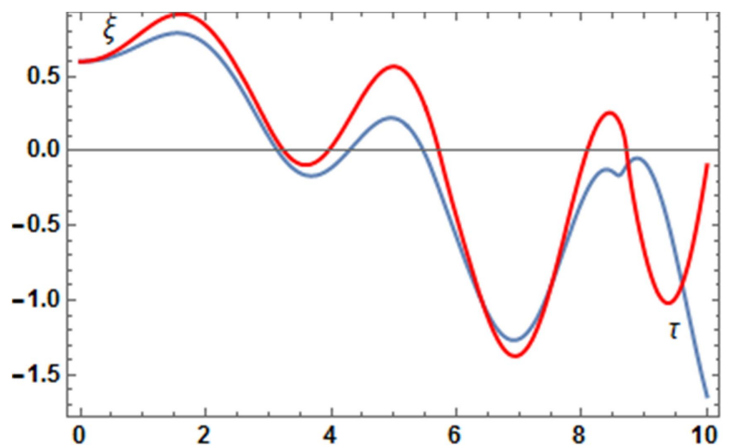

Fig.9: Time Series for $\sigma=0, \mathrm{q}_{1}=\{1$ (Magneta $\left.), 0.8(\operatorname{Red})\right\}$.

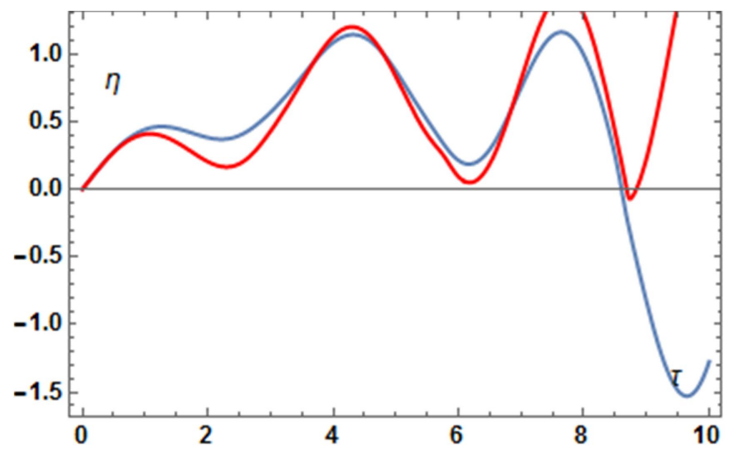

Fig.10: Time Series for $\sigma=0, \mathrm{q}_{1}=\{1$ (Magneta), $0.8($ Red $)\}$.

\subsection{Zero-velocity curves}

We have drawn the zero velocity curves around all the libration points for the fixed values of the parameters in both $\xi \eta$-plane (Figs. 11, 12, 13) and $\xi \zeta$ - plane (Fig.14, 15, 16).

i)

$$
(\xi, \eta) \text { - plane } \quad \alpha_{1}=0.2, \mathrm{k}=0.4, \nu=0.019,
$$

In this section, we have considered the oblateness $\sigma=0.1$, and solar radiation pressure $\mathrm{q}_{1}=1$, in this case, we have drawn seven zero velocity curves (Fig. 11) as Green at energy constant 1.67183, Blue at energy constant 1.19934, Purple at energy constant 1.4973, Magneta at energy constant 1.48138, Orange at energy constant 1.51643 , Cyan at energy constant 1.48517 , Black at energy constant 71.3618 (Fig. 12). Again when we have considered the oblateness $\sigma=0$, and solar radiation pressure $\mathrm{q}_{1}=0.8$, in this case, we have drawn five zero velocity curves (Fig. 13) as Green at energy constant 1.2374, Blue at energy constant 0.876046 , Purple at energy constant 1.20244, Magneta at energy constant 1.21705, Pink at energy constant 4.39616.

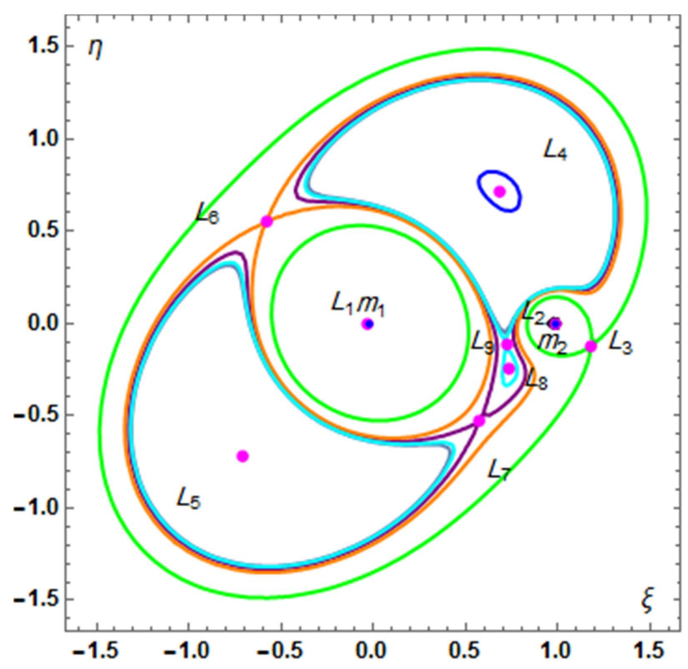

Fig.11: Zero velocity curves with oblateness for different seven values of energy constants

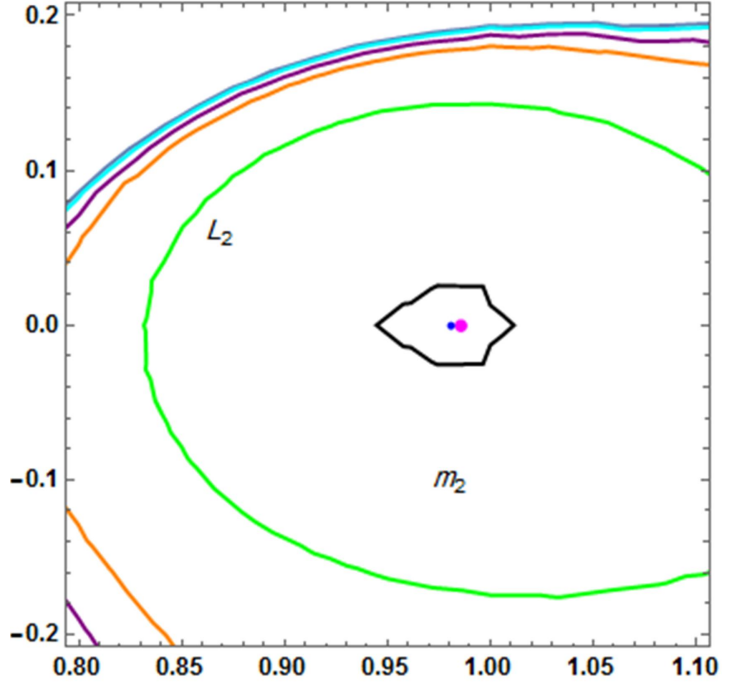

Fig. 12: The zoomed part of figure 11 near the primary $m_{2}$

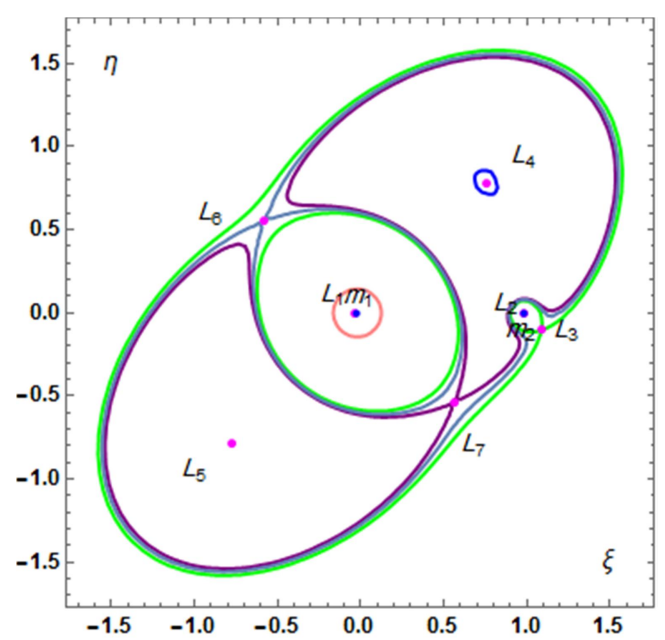

Fig.13: Zero velocity curves with radiation pressure for different five values of energy constants

ii)

$(\xi, \zeta)-$ plane $\quad \alpha_{1}=0.2, \mathrm{k}=0.4, v=0.019$,

In this section, we have considered the oblateness $\sigma=0.1$, and solar radiation pressure $\mathrm{q}_{1}=1$, in this case, we have drawn four zero velocity curves (Fig. 14) as Green at energy constant 1.63919 , Red at energy constant 1.38101, Blue at energy constant 1.47107, Black at energy constant 71.3618 (Fig. 15). Again when we have considered the oblateness $\sigma=0$, and solar radiation pressure $\mathrm{q}_{1}=0.8$, in this case, we have drawn four zero velocity curves (Fig. 16) as Green at energy constant 1.20889, Red at energy constant 1.07704, Blue at energy constant 1.12865, Black at energy constant 4.39616 . 

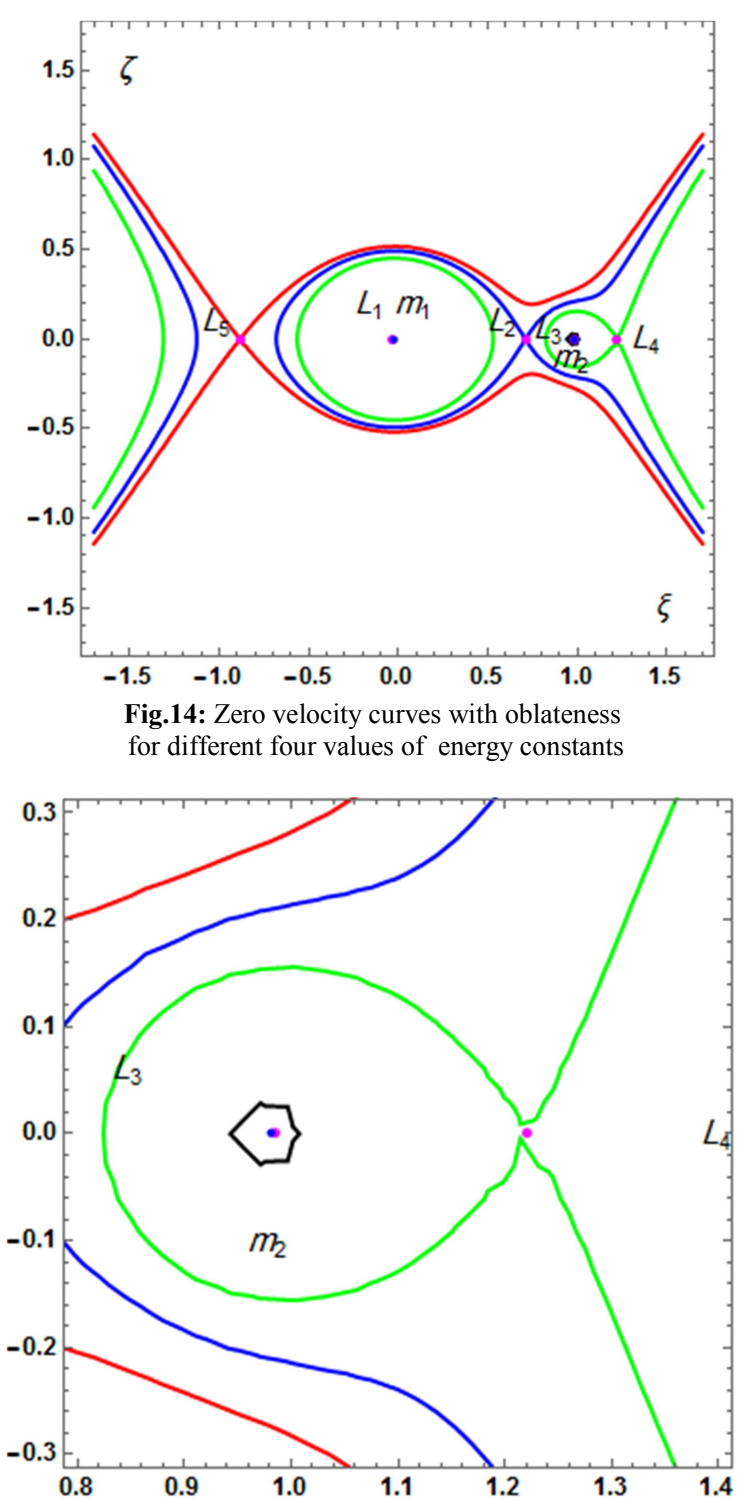

Fig.15: The zoomed part of figure 14 near the primary $\mathrm{m}_{2}$

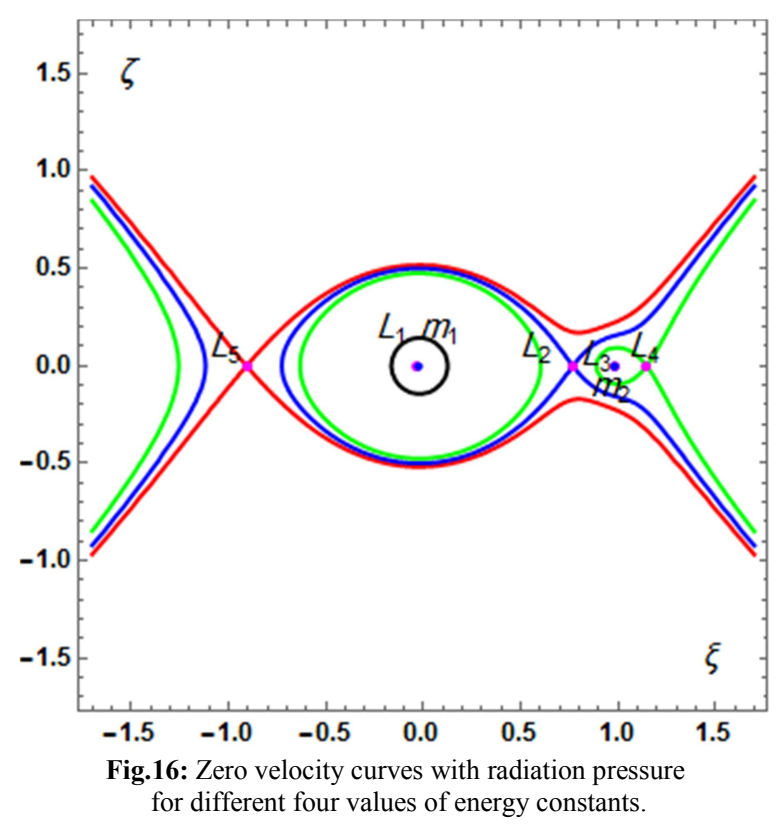

\subsection{Poincare surface of section}

We have also drawn the Poincare surface of sections with and without oblateness (Fig. 17) and observed that there is minor effect of oblateness and when we have drawn the Poincare surface of section with and without solar radiation pressure (Fig. 18), then we observed that the Poincare surface of sections are shrinking.

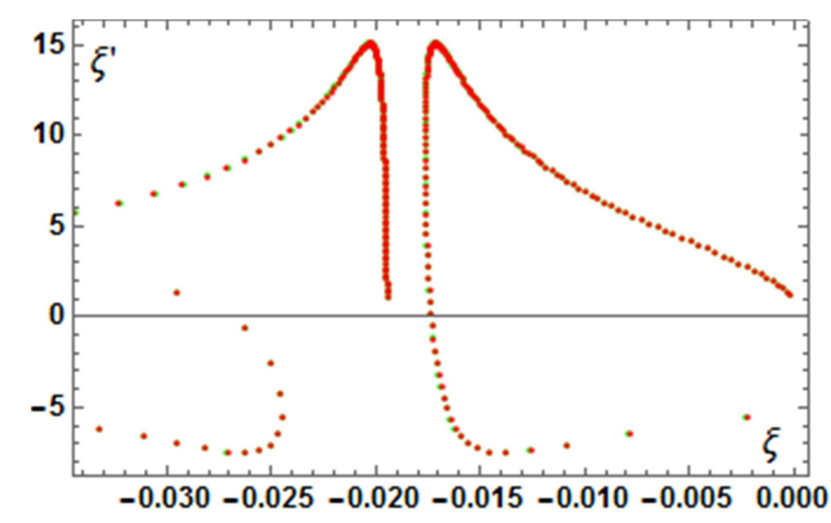

Fig.17: Poincare Surface of Sections $\sigma=0, \mathrm{q}_{1}=1($ Green $), \sigma=0.1, \mathrm{q}_{1}=1($ Red $)$.

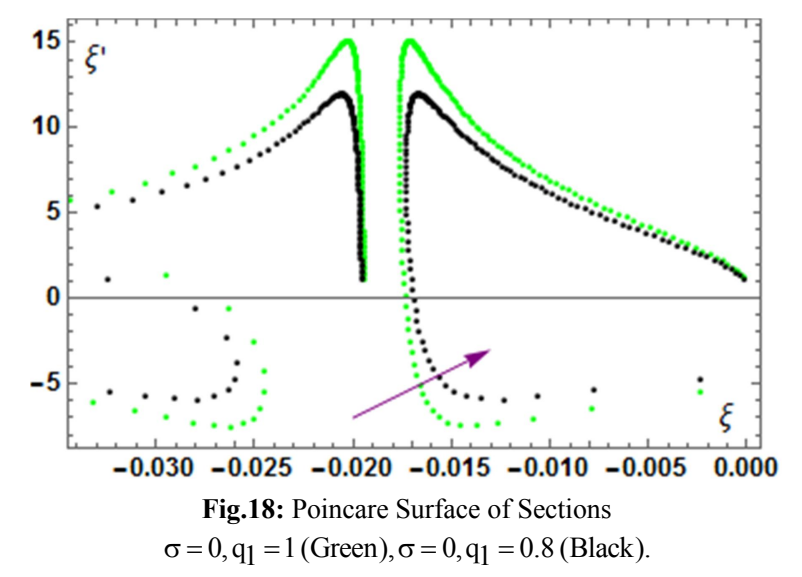

\section{Stability of the equilibrium points of the au- tonomized equations}

Following the procedure given by Mccuskey [17], we can examine the stability of the autonomized equations. When

$$
\xi=\xi_{0}+\mathrm{a}, \eta=\eta_{0}+\mathrm{b}, \zeta=\zeta_{0}+\mathrm{c}
$$

As

$$
\begin{aligned}
& \mathrm{a}^{\prime \prime}-2 \omega_{0} \mathrm{~b}^{\prime}-\alpha_{1} \mathrm{a}^{\prime}=\mathrm{a}\left(\Omega_{\xi \xi}\right)_{0}+\mathrm{b}\left(\Omega_{\xi \eta}\right)_{0}+\mathrm{c}\left(\Omega_{\xi \zeta}\right)_{0}, \\
& \mathrm{~b}^{\prime \prime}+2 \omega_{0} \mathrm{a}^{\prime}-\alpha_{1} \mathrm{~b}^{\prime}=\mathrm{a}\left(\Omega_{\eta}\right)_{0}+\mathrm{b}\left(\Omega_{\eta \eta}\right)_{0}+\mathrm{c}\left(\Omega_{\eta \zeta}\right)_{0}, \\
& \mathrm{c}^{\prime \prime}-\alpha_{1} \mathrm{c}^{\prime}=\mathrm{a}\left(\Omega_{\zeta \xi}\right)_{0}+\mathrm{b}\left(\Omega_{\zeta \eta}\right)_{0}+\mathrm{c}\left(\Omega_{\zeta \zeta}\right)_{0},
\end{aligned}
$$

Where $a, b$ and $c$ are the small displacements of the infinitesimal body from the libration point. Suffix zero denotes the value at the libration point.

To solve equation (7), let $\mathrm{a}=\mathrm{Ae}^{\lambda \tau}, \mathrm{b}=\mathrm{Be}^{\lambda \tau}, \mathrm{c}=\mathrm{Ce}^{\lambda \tau}$, where $\mathrm{A}, \mathrm{B}$ and $\mathrm{C}$ are parameters.

Substituting these values in equation (7) and rearranging, we get 


$$
\begin{aligned}
& \mathrm{A}\left(\lambda^{2}-\alpha_{1} \lambda-\left(\Omega_{\xi \xi}\right)_{0}\right)-\mathrm{B}\left(2 \omega_{0} \lambda+\left(\Omega_{\xi \eta}\right)_{0}\right)-\mathrm{C}\left(\Omega_{\xi \zeta}\right)_{0}=0, \\
& \mathrm{~A}\left(2 \omega_{0} \lambda-\left(\Omega_{\eta}\right)_{0}\right)+\mathrm{B}\left(\lambda^{2}-\alpha_{1} \lambda-\left(\Omega_{\eta \eta}\right)_{0}\right)-\mathrm{C}\left(\Omega_{\eta}\right)_{0}=0, \\
& -\mathrm{A}\left(\Omega_{\zeta \xi}\right)_{0}-\mathrm{B}\left(\Omega_{\zeta \eta}\right)_{0}+\mathrm{C}\left(\lambda^{2}-\alpha_{1} \lambda-\left(\Omega_{\zeta \zeta}\right)_{0}\right)=0,
\end{aligned}
$$

The equation (8) will have a non-trivial solution for $\mathrm{A}, \mathrm{B}$ and $\mathrm{C}$ if

$$
\begin{aligned}
& \left|\begin{array}{ccc}
\lambda^{2}-\alpha_{1} \lambda-\left(\Omega_{\xi \xi}\right)_{0} & -\left(2 \omega_{0} \lambda+\left(\Omega_{\xi \eta}\right)_{0}\right) & -\left(\Omega_{\xi \zeta}\right)_{0} \\
2 \omega_{0} \lambda+\left(\Omega_{\eta \xi}\right)_{0} & \lambda^{2}-\alpha_{1} \lambda-\left(\Omega_{\eta \eta}\right)_{0} & -\left(\Omega_{\eta \zeta}\right)_{0} \\
-\left(\Omega_{\zeta \xi}\right)_{0} & -\left(\Omega_{\zeta \eta}\right)_{0} & \lambda^{2}-\alpha_{1} \lambda-\left(\Omega_{\zeta \zeta}\right)_{0}
\end{array}\right|=0, \\
& \lambda^{6}-3 \alpha_{1} \lambda^{5}+\lambda^{4}\left(4 \omega_{0}^{2}+3 \alpha_{1}^{2}-\left(\Omega_{\xi \xi}\right)_{0}-\left(\Omega_{\eta \eta}\right)_{0}-\left(\Omega_{\zeta \zeta}\right)_{0}\right) \\
& +\alpha_{1} \lambda^{3}\left(-4 \omega_{0}^{2}-\alpha_{1}^{2}+2\left(\Omega_{\xi \xi}\right)_{0}+2\left(\Omega_{\eta \eta}\right)_{0}+2\left(\Omega_{\zeta \zeta}\right)_{0}\right) \\
& +\lambda^{2}\left(-\left(\Omega_{\xi \eta}\right)_{0}^{2}-\left(\Omega_{\xi \zeta}\right)_{0}^{2}+\left(\Omega_{\xi \xi}\right)_{0}\left(\Omega_{\eta \eta}\right)_{0}-\left(\Omega_{\eta \zeta}\right)_{0}^{2}-\right. \\
& 4 \omega_{0}^{2}\left(\Omega_{\zeta \zeta}\right)_{0}+\left(\Omega_{\xi \xi}\right)_{0}\left(\Omega_{\zeta \zeta}\right)_{0}+\left(\Omega_{\zeta \zeta}\right)_{0}\left(\Omega_{\eta \eta}\right)_{0}-\alpha_{1}^{2}\left(\Omega_{\xi \xi}\right)_{0} \\
& \left.-\alpha_{1}^{2}\left(\Omega_{\eta \eta}\right)_{0}-\alpha_{1}^{2}\left(\Omega_{\zeta \zeta}\right)_{0}\right)+\alpha_{1} \lambda\left(\left(\Omega_{\xi \eta}\right)_{0}^{2}+\left(\Omega_{\xi \zeta}\right)_{0}^{2}\right. \\
& \left.-\left(\Omega_{\xi \xi}\right)_{0}\left(\Omega_{\eta \eta}\right)_{0}+\left(\Omega_{\eta \zeta}\right)_{0}^{2}-\left(\Omega_{\xi \xi}\right)_{0}\left(\Omega_{\zeta \zeta}\right)_{0}-\left(\Omega_{\eta \eta}\right)_{0}\left(\Omega_{\zeta \zeta}\right)_{0}\right) \\
& +\left(\left(\Omega_{\xi \zeta}\right)_{0}^{2}\left(\Omega_{\eta \eta}\right)_{0}-2\left(\Omega_{\xi \eta}\right)_{0}\left(\Omega_{\xi \zeta}\right)_{0}\left(\Omega_{\eta \zeta}\right)_{0}+\left(\Omega_{\xi \xi}\right)_{0}\left(\Omega_{\eta \zeta}\right)_{0}^{2}\right. \\
& \left.+\left(\Omega_{\xi \eta}\right)_{0}^{2}\left(\Omega_{\zeta \zeta}\right)_{0}-\left(\Omega_{\xi \xi}\right)_{0}\left(\Omega_{\eta \eta}\right)_{0}\left(\Omega_{\zeta \zeta}\right)_{0}\right)=0,
\end{aligned}
$$

Here, we have examined the stability of the libration points by finding the characteristic roots of equation (9) numerically. We have calculated the characteristic roots for the different values of the libration points at $\alpha_{1}=0.2, \mathrm{k}=0.4, v=0.019$ and observed that in all the cases, there exists at least one positive real characteristic root. Hence, it can be concluded that all the libration points are unstable.

\section{Conclusion}

Here, we have investigated the dynamics in the restricted problem by considering one of the primaries as oblate and other one as solar radiation pressure and all the masses are variable (primaries as well as infinitesimal body). We have determined the autonomous equations of motion which is different from the equations of motion found with only infinitesimal variable mass by the factors $\sigma, q_{1}, \alpha_{1}$. And in the next numerical analysis section, we have drawn libration points (Figures 2, 3, 4, 5, 6), the time series (Figures $7,8,9,10$ ), the zero velocity curves (Figures 11, 12, 13, 14, 15,16 ), the Poincare surface of sections (Figures 17, 18) with the effects of oblateness and solar radiation pressure. And observed that the libration points are moving towards the origin, the time series are shifting by some phase angles, and the Poincare surface of sections are shrinking due to the perturbations. Finally we have examined the stability for each libration points and found that all the libration points are unstable.

\section{Acknowledgement}

We are thankful to the Deanship of Scientific Research, College of Science in Zulfi, Majmaah University, Kingdom of Saudi Arabia, for providing all the research facilities in the completion of this research work.

\section{References}

[1] Meshcherskii, I. V., "Works on the Mechanics of Bodies of Variable Mass", GITTL, Moscow, 1952.

[2] Szebehely, V., "Theory of Orbits", Academic Press, New York, 1967.

[3] Chernikov, Yu. A., "The photogravitational restricted three body problem", Soviet Astron. A. J., vol. 14, No. 1, pp. 176-181, 1970.

[4] Schuerman, D.W.,"Roche Potentials including radiation effects", Astrophysics and space science, vol. 19, pp. 351-358, 1972. https://doi.org/10.1007/BF00645718.

[5] Subbarao, P. V., Sharma, R. K.,"A note on the stability of the triangular points of equilibrium in the restricted three body problem", Astron. \& Astrophys. 43, 381-383, 1975.

[6] Bhatnagar, K. B., Chawla, J.M.,"The effect of oblateness of the bigger primary on the collinear libration points in the restricted problem of three bodies", Celest. Mech., vol. 16, pp. $129-136$, 1977. https://doi.org/10.1007/BF01228595.

[7] Bhatnagar, K. B., Hallan, P.P., "Effect of perturbed potentials on the stability of libration points in the restricted problem", Celest. $\begin{array}{lllll}\text { Mech. } & \text { vol. } & \text { 20, } & \text { 95-103, } & \end{array}$ https://doi.org/10.1007/BF01230231.

[8] Simmons, J.F.L., Mcdonald, A.J.C., Brown, J.C.,"The restricted 3body problem with radiation pressure" Celest. Mech. vol. 35, pp. 145-187, 1985. https://doi.org/10.1007/BF01227667.

[9] Singh, J., Ishwar, B.,"Stability of triangular points in the generalized photogravitational restricted three body problem", Bull. Astro. Sco. India, vol. 27, pp. 415-424, 1999.

[10] AbdulRaheem, A. R., Singh, J., "Combined effects of perturbations, radiation and oblateness on the stability of equilibrium points in the restricted three body problem," The Astronomical Journal, 131, 1880-1885, 2006. https://doi.org/10.1086/499300.

[11] Singh, J., Leke, O.," Stability of the photogravitational restricted three-body problem with variable masses," Astrophys Space Sci (2010) 326: 305-314, https://doi.org/10.1007/s10509-009-0253-x.

[12] Singh, J., Sandah, A.U., " Existence and Linear Stability of Equilibrium Points in the Robe's Restricted Three-Body Problem with Oblateness", Hindawi Publishing Corporation Advances in Mathematical Physics, vol. 2012, Article ID 679063, 18 pages https://doi.org/10.1155/2012/679063.

[13] Abouelmagd, E.I., El-Shaboury, S.M.," Periodic orbits under combined effects of oblateness and radiation in the restricted problem of three bodies," Astrophys Space Sci., 341:331-341, 2012, https://doi.org/10.1007/s10509-012-1093-7.

[14] E. I. Abouelmagd, E. I.,J.L.G. Guirao, J " The effect of oblateness in the perturbed restricted three-body problem", Meccanica, 48, 2479-2490, 2013, https://doi.org/10.1007/s11012-013-9762-3.

[15] Abdullah, "Stability of the equilibrium points in the circular restricted four body problem with oblate primary and variable mass," International Journal of Advanced Astronomy, vol. 4, no. 1, pp. 1419, 2016. https://doi.org/10.14419/ijaa.v4i1.5831.

[16] Mittal, A., et. al., "Stability of libration points in the restricted fourbody problem with variable mass," Astrophysics and space science, 361, 329, 2016, https://doi.org/10.1007/s10509-016-2901-2.

[17] Mccuskey, S. W., "Introduction to Celestial Mechanics," AddisonWesley, USA, 1963. 